\title{
Multi-site, multi-colour and space photometry of a southern $\delta$ Scuti star, 38 Eri
}

\author{
Margit Paparó ${ }^{1, \star}$, József Benkö ${ }^{1}$, and ground-based and MOST photometry teams \\ ${ }^{1}$ Konkoly Observatory, MTA CSFK, Konkoly Thege u 15-17, H-1125 Budapest, Hungary
}

\begin{abstract}
Eri is a $\delta$ Scuti star in the shell hydrogen burning stage of evolution, with a 0.04 mag peak-to-peak amplitude and a very complex light variation. It was discovered in 1971. In the subsequent investigation the star always showed a different frequency content. A ground-based multi-site campaign and a MOST observation were devoted to resolve the contradicting pulsational behaviour. We present the preliminary result of our investigation obtained on Strömgren $b$ and MOST data. The complexity of the pulsation is confirmed, but the comprehensive analysis helps to resolve the frequencies of the fast rotating star.
\end{abstract}

\section{Introduction}

All the previous ground-based observations, both photometric and spectroscopic, revealed a very complex frequency content of 38 Eri. None of them confirmed the previous set of frequencies, some frequencies were missing or new ones appeared. It was questionable that the excited amplitudes of the frequencies were changing or the frequency content was not properly resolved. A ground-based multi-site campaign and the MOST space data were devoted to answer the question.

\section{Single site results: effect of different sampling}

The preliminary analyses revealed how serious a task it is to find the frequencies using single site data. A basic problem is the daily alias structure. Additionally, the rotational splitting due to the fast rotation plays an important role. The different subsets of the ground-based campaign show the serious effect of the different sampling. The left and right panels of Figure 1 show the variety of the frequencies that we obtained for two different segments. The dominant frequencies, around $13.3 \mathrm{~d}^{-1}$, are the same in both datasets, but the subsequent frequencies in the prewhitening process are different. We can recognize a doublet (left panel) or triplet (right panel) at around $10.47 \mathrm{~d}^{-1}$, but the peaks around $5.2 \mathrm{~d}^{-1}$ appear only in the dataset with better sampling. An example for the possible confusion between the $1 \mathrm{~d}^{-1}$ alias and the independent frequency is the two peaks at 9.47 and $10.47 \mathrm{~d}^{-1}$. A peak at $12.62 \mathrm{~d}^{-1}$ appears only in the subset with better sampling. The preliminary investigation confirms that the single site observation is not enough for resolving the complexity of the pulsation in 38 Eri.

^paparo@konkoly.hu 

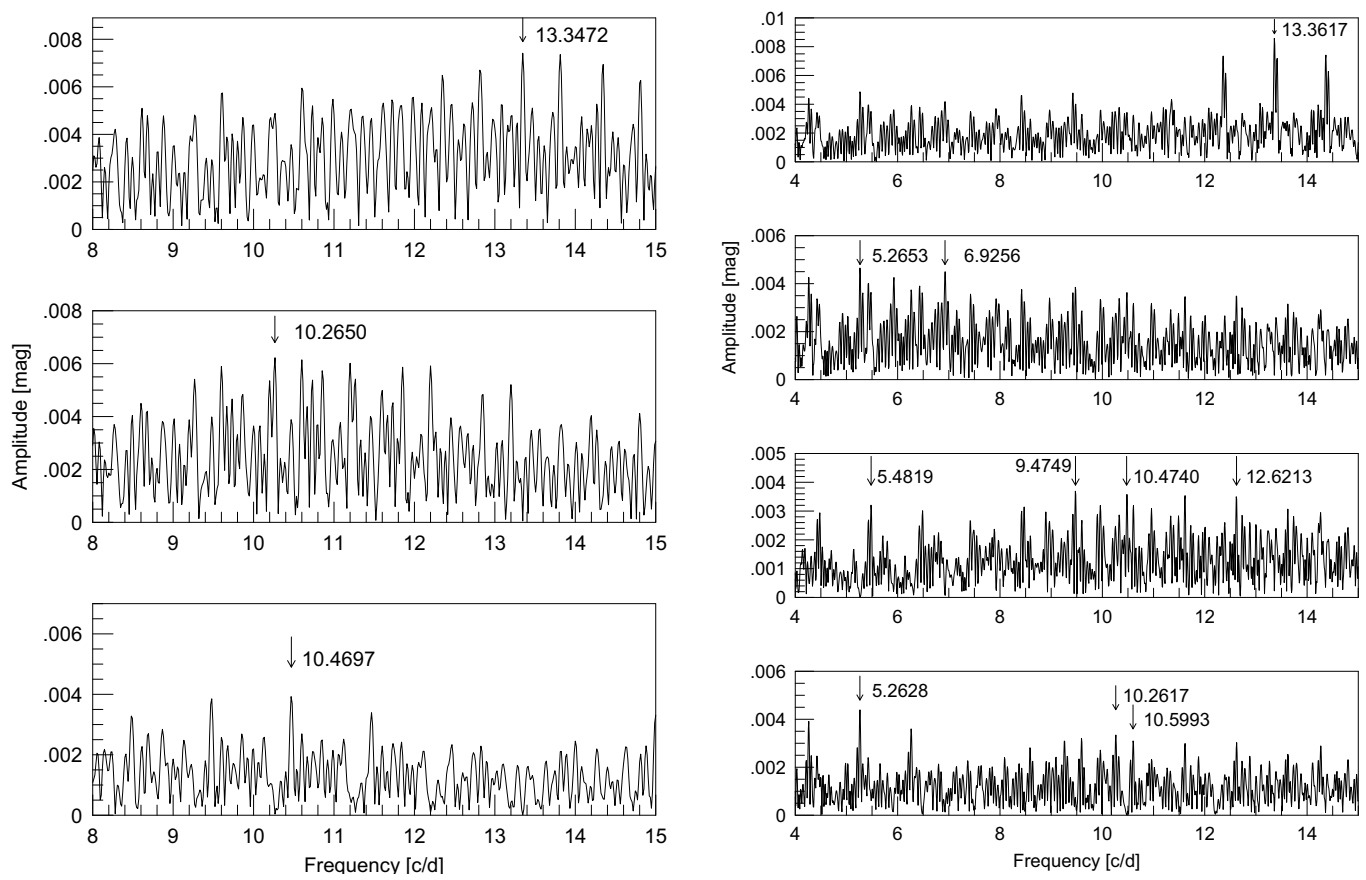

Figure 1. Left panel: the original spectrum and two pre-whitened spectra of one segment of the ground-based campaign are shown. Right panel: Another set of the ground-based campaign represents a different sampling. Some distinguished spectra are shown.

\section{MOST results}

The $\delta$ Scuti star 38 Eri was a switch target with two other targets, so only part of each MOST 101minute orbit was devoted to it. This is a regular sampling along the orbital period, but it results in an irregular sampling along the light curve. The effect of the different sampling may be followed in the MOST data, too. Due to the larger timebase we can resolve the frequencies with even lower separation. Dividing the whole dataset into two subsets, different frequencies dominate the frequency spectrum of the subsets. It is suggested that the sampling influences not only the frequency content, but the amplitudes, too.

\section{Conclusion}

Each previous investigation of the $\delta$ Scuti star 38 Eri could catch part of the complexity of the pulsation. The amplitude of the excited modes seems to vary from epoch to epoch resulting in different modes being dominant at a certain epoch. The final analysis may find the whole set of frequencies and lead to the localization of the rotational triplets of the non-radial modes.

Acknowledgments: This work was supported by the grant: ESA PECS No 4000103541/11/NL/KML. 